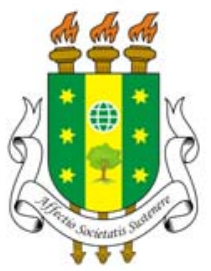

SRBBADM

Journal homepage:

www.arvore.org.br/seer

\section{UMA PROPOSTA PARA ANÁLISE DA ADOÇÃO DE TECNOLOGIAS DA INFORMAÇÃO EM MICRO E PEQUENAS EMPRESAS A PARTIR DA ADAPTAÇÃO DO MODELO TOE (TECHNOLOGY, ORGANIZATION AND ENVIRONMENT)}

\section{RESUMO}

Este ensaio teórico tem como objetivo propor a adaptação do modelo TOE Technology, Organization and Environment para análise da adoção de tecnologias da informação em micro e pequenas empresas. Este modelo tem sido testado e validado por vários estudos e é considerado um modelo aplicável à adoção $e$ difusão de tecnologia, ele argumenta que o processo pelo qual uma organização adota e implementa inovações tecnológicas é influenciado pelo contextos tecnológico, organizacional e ambiental. A intenção propositiva deste estudo tem início a partir da reformulação do aspecto central do modelo TOE, ou seja, o que é colocado originalmente como tomada de decisão para inovação tecnológica, terá um direcionamento específico para tomada de decisão no âmbito da adoção de tecnologia da informação. Além disso, o modelo aqui proposto busca também respaldo nos estudos sobre processo decisório para que possa contribuir para a evolução do entendimento teórico e empírico relativo à adoção de TI. Nesse sentido, o presente ensaio busca evidenciar a relevância do tema e do objeto de análise, tanto a partir da perspectiva teórica quanto da pragmática, já que são organizações essenciais do ponto de vista econômico e social. A partir da proposição, sugere-se que pesquisas futuras venham a aplicar o modelo para que se verifique a riqueza exploratória de análise em organizações, principalmente as MPE.

PALAVRAS-CHAVES: Adoção de Tecnologias da Informação; TOE; Technology, Organization and Environment; Micro e Pequenas Empresas.

\section{A PROPOSAL FOR ANALYZING THE ADOPTION OF INFORMATION TECHNOLOGIES IN MICRO AND SMALL ENTERPRISES FROM THE ADAPTATION OF THE MODEL TOE (TECHNOLOGY, ORGANIZATION AND ENVIRONMENT)}

\section{ABSTRACT}

This theoretical essay aims to propose an adaptation of the TOE - Technology, Organization and Environment - model to analyze Information Technology adoption in micro and small enterprises. This model has been tested and validated by several studies and is considered a model applicable to the adoption and dissemination of technology, he argues that the process by which an organization adopts and implements technological innovations is influenced by technological contexts, organizational and environmental. The purposeful intention of this study starts from the reformulation of the central aspect of the model TOE, in other words, which is originally placed as decision making for technological innovation, have a specific direction for decision making within the adoption of information technology Moreover, the proposed model also seeks support in studies on decision making so that it can contribute to the development of theoretical and empirical understanding concerning the adoption of IT. Accordingly, this essay seeks to provide evidence for the relevance of the subject and the object for analysis, from both theoretical and pragmatic perspectives, since these organizations are economically and socially essential. Starting with that proposition, it suggests future research that could apply the model to verify the exploratory richness of organizational analysis, principally for micro and small enterprises.

KEYWORDS: Information Technology Adoption; TOE; Technology, Organization and Environment; Micro and Small Enterprises.
Revista Brasileira de Administração Científica, Aquidabã, v.4, n.2, Ago 2013.

\section{ISSN 2179-684X}

\section{SECTION: Articles}

TOPIC: Sistemas e Tecnologia da Informação

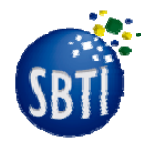

Anais do Simpósio Brasileiro de Tecnologia da Informação (SBTI 2013)

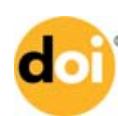

DOI: 10.6008/ESS2179-684X.2013.002.0018

Rodrigo Cesar Reis de Oliveira

Universidade Federal da Bahia, Brasil http://lattes.cnpq.br/2448160679532667 rodrigopesquisando@gmail.com

\section{Ernani Marques Santos}

Universidade Federal da Bahia, Brasil http://lattes.cnpq.br/5388965130432483 ernanims@gmail.com

Ivo Pedro Gonzalez Júnior Universidade Federal da Bahia, Brasil http://lattes.cnpq.br/9172835049817642 ivojunior@gmail.com

Received: 07/07/2013

Approved: 05/08/2013

Reviewed anonymously in the process of blind peer.

\section{Referencing this:}

OLIVEIRA, R. C. R.; SANTOS, E. M.; JÚNIOR, I. P. G.. Uma proposta para análise da adoção de tecnologias da informação em micro e pequenas empresas a partir da adaptação do modelo TOE (Technology, Organization and Environment). Revista Brasileira de Administração Científica, Aquidabã, v.4, n.2, p.257-272, 2013. DOI: http://dx.doi.org/10.6008/ESS2179- 


\section{INTRODUÇÃO}

A tecnologia da informação ( $\mathrm{TI}$ ) tem sido adotada nos mais diversos ambientes organizacionais. De fato, ela está presente e contribui para a busca de vantagem competitiva dos negócios. Audy e Brodbeck (2003) argumentam que sua efetiva utilização é crucial e os investimentos em TI devem ser feitos e alinhados à estratégia da empresa.

Os resultados obtidos a partir da TI se devem não só à tecnologia isoladamente, mas a forma como a mesma é incorporada. Nesse sentido, as organizações devem perceber a importância do processo de adoção de tecnologias da informação (ADTI). Assim, poderão planejar e entender tal processo para que obtenham sucesso com novas aquisições.

Os bons resultados dos negócios creditados ao uso da TI não dependem apenas do investimento nela realizado, mas de como tal investimento é planejado e da forma como a tecnologia é implantada na organização. Quando bem gerenciada, a tecnologia possibilita para as organizações a penetração em novos mercados e a agregação de valor. Assim, empresas já não podem pensar em projetos de negócio ou na formulação de estratégias sem considerar a importância da TI (TAPSCOTT, 1999).

De acordo com Meirelles (2003), o percentual da receita líquida para investimentos em informática passa a figurar como fator estratégico para o sucesso de empresas. Assim, o aumento do volume investido deve pressupor maior expertise acerca da escolha dos tipos e volumes de tecnologias a serem adquiridos. Com isso, fica evidente o papel relevante que a tecnologia tem desempenhado dentro das organizações.

Diante de tal relevância, um dos aspectos que permeiam as organizações é a questão da adoção de tecnologia da informação, já que a constante e rápida evolução dos recursos tecnológicos representa considerável aumento da diversidade de opções a serem adotadas. Desse modo, gestores precisam monitorar continuamente o ambiente, além de verificar novas alternativas tecnológicas. Assim, é possível descobrir ferramentas de $\mathrm{Tl}$ que possam ser incorporadas ou mesmo substituir as utilizadas atualmente no ambiente organizacional. Ademais, empresários devem avaliar e decidir qual a melhor opção para adaptar à necessidade do negócio. Contudo, é preciso perceber que o processo de ADTI não é trivial, pois envolve decisões complexas, arriscadas e incertas, com pouca ou nenhuma garantia de sucesso (SUÁREZ; SILVA; SOUZA, 2011).

A essencialidade das $\mathrm{TI}$ pode ser evidenciada a partir dos investimentos realizados para aquisição das mesmas. Os gastos são crescentes e pesquisas mostram que as organizações brasileiras irão investir 133,9 bilhões de dólares em TI em 2013, o que representa um aumento de $6 \%$ se comparado com a estimativa para 2012, de 126,3 bilhões de dólares (COMPUTERWORLD, 2012).

De acordo com Lunardi, Dolci e Maçada (2010) é crescente e evidente o aumento do uso de $\mathrm{TI}$ também em micro e pequenas empresas (MPE), principalmente por fatores como a 
diminuição dos custos de aquisição, a busca por vantagem competitiva, além de exigências legais e de parceiros de negócio. Entretanto, pouca literatura referente à utilização de TI nessas empresas tem sido encontrada. Constatação agravada quando percebe-se ainda menor incidência de estudos em países como o Brasil.

O crescimento da indústria mundial de software, segundo Guerra, Capovilla e Thienne (2005), ocorre em uma velocidade jamais vista em outro setor. Tal aceleração da oferta é pressionada pelo aumento da demanda por produtos de tecnologia da informação, tanto na esfera individual quanto organizacional. Assim, visto o crescimento da aquisição, é essencial que estudos sejam conduzidos para o entendimento desse fenômeno, que necessariamente perpassa o entendimento dos processos de adoção das $\mathrm{TI}$, tanto entre consumidores quanto empresas.

Tais processos, em particular os relacionados às MPE, devem ser conhecidos e analisados para que empresas dessa natureza possam enveredar pela busca consistente de vantagem competitiva. Muitas das oportunidades, problemas e assuntos gerenciais relacionados às empresas de pequeno porte, principalmente os relativos a TI, são únicos e como tal, merecem pesquisas específicas que tentem preencher lacunas de entendimento (LUNARDI; DOLCl; MAÇADA, 2010).

Ao tratar das especificidades inerentes às MPE, no que tange o uso da TI, Ramos, Silva e Alvarega (2009) consideram que o uso adequado e eficaz de tecnologia da informação só é possível se a aquisição de tais recursos for planejada. Concomitantemente, proprietários e principais usuários devem ter conhecimento dos processos de adoção de $\mathrm{Tl}$ e das mudanças inerentes e necessárias, bem como dos potenciais e das limitações que podem aflorar das tecnologias e das pessoas.

De fato, grande parte da literatura encontrada sobre adoção de TI concorda com a relevância do entendimento apropriado do tema, entretanto, há poucos estudos de comparação entre os modelos teóricos de ADTI, no nível individual e ainda menos esforços teórico-empíricos no nível de análise organizacional (OLIVEIRA; MARTINS, 2011). Assim, o esforço aqui proposto se justifica quando lança-se à busca de entendimento da adoção de TI entre as micro e pequenas empresas, com o intento de proposição de modelo.

Ademais, este ensaio ancora sua relevância na perspectiva propositiva de análise da adoção de $\mathrm{TI}$ a partir do modelo technology, organization and enviroment (TOE) que aqui será adaptada sob influência dos conceitos de processo decisório, diante da aparente falta de estudo dessa natureza. Dentro do contexto das diversas nuances relativas à adoção de TI, Santos (2005) afirma que tal processo exige que se tomem decisões de aquisição num contexto de riscos e incertezas, diante de tamanha diversidade de alternativas existentes em termos de tecnologia, padrões tecnológicos e fornecedores. Ainda segundo esse autor, a TI se apresenta como um dilema aos gestores que, de um lado, têm a TI como recurso essencial para obtenção de diferencial competitivo e, de outro, a velocidade das mudanças tecnológicas torna complexo o processo de tomada de decisão inerente a TI. 
A dinamicidade de tais mudanças tecnológicas tem afetado inclusive a sobrevivência das MPE. Segundo Santos Júnior, Freitas e Luciano (2005), essas empresas são notadamente relevantes, pois geram emprego e renda para boa parte da população do Brasil. Entretanto, o pequeno porte traz também dificuldades e vulnerabilidades aos riscos de mercado. Tal problemática pode ser ilustrada a partir da verificação que essas empresas possuem carência de recursos, dificuldade de sobrevivência no mercado e, geralmente, apresentam barreiras fracas a novos entrantes, baixo poder de barganha com fornecedores e clientes e os produtos e serviços são passíveis de substituição, tornando o ambiente altamente competitivo.

Nas últimas décadas cresceu a representatividade tanto da TI quanto das MPE, tanto no âmbito social quanto econômico, como pode ser visto no quadro 1, sobre os dados gerais das MPE brasileiras.

Quadro 1: Dados gerais das MPE brasileiras.

\begin{tabular}{|c|c|}
\hline As MPE no Brasil & O que isso representa \\
\hline $20 \%$ do PIB & R $\$ 700$ bilhões \\
\hline $99 \%$ das empresas & 5,7 milhões de MPE \\
\hline $60 \%$ dos empregos & 56,4 milhões de empregos \\
\hline
\end{tabular}

Fonte: SEBRAE (2012).

Nesse contexto, o presente estudo busca contribuir para um melhor entendimento, sistematização e geração de conhecimento acerca do tema ADTI, por meio da proposição de uma adaptação do modelo TOE para uso na análise do processo de adoção de TI por MPE. Desse modo, espera-se contribuir também para o desenvolvimento de processos empresariais mais adequados a adoção de $\mathrm{TI}$, para que estas sejam cada vez mais alinhadas com as necessidades e estratégias organizacionais.

O presente ensaio está estruturado em seções que buscam evidenciar a relevância do estudo para a análise dos processos de adoção de tecnologias da informação em micro e pequenas empresas. Na seção inicial, é feita esta introdução, no referencial teórico são trabalhados aspectos essenciais ao tema e ao modelo proposto. Por fim, são apresentadas as considerações finais e as referências usadas no presente estudo.

\section{METODOLOGIA}

O presente estudo é um ensaio teórico, tendo como base publicações nacionais e internacionais para atingir o objetivo propositivo de adaptação do modelo TOE - Technology, Organization and Environment. Este modelo, proposto no início dos anos 90, deu início a um novo tipo de pesquisa sobre difusão tecnológica, com foco no processo de inovação organizacional. De acordo com Tornatzky e Fleischer (1990), há três elementos do contexto de uma empresa que influenciam o processo através do qual esta adota e implementa inovações tecnológicas: contexto organizacional, contexto tecnológico e contexto ambiental. 
Vale salientar que Tornatzky e Fleischer (1990), apresentaram o modelo de inovação tecnológica em que a organização, a tecnologia e o meio ambiente externo fazem parte de um esquema que envolve o processo de tomada de decisão para inovação tecnológica. Utilizando este modelo como base, adaptou-se o mesmo, atualizando-o em função da evolução dos processos organizacionais, principalmente aqueles ligados à adoção de tecnologias da informação. Ademais, busca-se considerar a relevância social e econômica das Micro e Pequenas Empresas (MPE) que possuem pouca atenção dos estudos acadêmicos dessa natureza. Assim, o foco foi propor a renovação do TOE para análise da adoção de tecnologias da informação em micro e pequenas empresas.

Adiciona-se ao modelo críticas sobre a falta de estudos que contribuam para a evolução do modelo TOE. Desse modo, como ponto de partida foi analisada a reformulação do aspecto central do modelo, a tomada de decisão para inovação tecnológica, tendo um direcionamento específico para tomada de decisão no âmbito da adoção de tecnologia da informação para micro e pequenas empresas.

\section{DISCUSSÃO TEÓRICA}

\section{Tecnologia da Informação}

A importância da tecnologia da informação tem sido atribuída para os diferentes tipos de empresas, independente do seu porte ou ramo de negócio. Torna-se não só um fator estratégico, mas essencialmente de sobrevivência das empresas em mercados progressivamente competitivos. Dessa forma, o uso dessas tecnologias tem sido objeto de preocupação e investigação de diversos segmentos, perpassando fortemente os ambientes empresariais e acadêmicos (ALBANO, 2000).

A TI pode ser entendida como a combinação estruturada de hardware, software e redes de computadores, usados na execução de tarefas de processamento de dados, tais como: captura, transmissão, estocagem, recuperação, manipulação e exibição de dados (CORDENONSI, 2005). Tais tarefas têm sido essenciais às organizações, desde processos operacionais aos estratégicos, contribuindo para melhoria dos mesmos e para tomada de decisões mais consistentes, principalmente quando a TI é usada via sistemas de informação.

Percebe-se um crescimento quantitativo e qualitativo na absorção da TI nas unidades de negócio. De fato, a evolução contínua das TI tem influenciado as possibilidades de inovação dos negócios. Para Batista (2004), a implantação de tecnologias da informação pode resultar em sucesso apenas se os gestores souberem lidar com três perspectivas que são inerentes ao contexto organizacional: organização, tecnologia e pessoas.

O impacto da implantação da TI pode ser evidenciado pelo argumento de Amaral Filho (2012), quando afirma que a década de 1990 assistiu ao uso intensivo das TI, por indivíduos, 
empresas e governos em suas rotinas de atividades. Isso implicou na troca de informações e imagens através das redes de computadores que gerou a relativização das distâncias espaciais. Desse modo, emerge um novo conceito, o da proximidade organizacional, viabilizado pela inserção de indivíduos e organizações nas redes de comunicação.

\section{Adoção de Tecnologia da Informação}

Apesar do aumento evidente dos investimentos em TI no Brasil, ainda existem dúvidas entre pesquisadores e executivos sobre os tipos de benefícios proporcionados por ela. Nesse sentido, Beltrame e Maçada (2009) argumentam que a TI não é apenas uma ferramenta para automatização de processos existentes, mas também um facilitador das mudanças organizacionais que podem conduzir a organização a ganhos adicionais de produtividade.

Suárez, Silva e Souza (2011) complementam os argumentos dos autores supracitados e afirmam que o papel das TI tem mudado, a partir do cenário global inerente aos negócios. Passam então a representar dispositivos estratégicos e competitivos de transformação das estruturas, processos e estratégias organizacionais. Desse modo, o processo de adoção de tecnologias da informação é considerado complexo, tornando-se necessária a adoção de ferramentas para auxiliar os gestores na avaliação do investimento em TI, aumentando a transparência da decisão em relação à adoção, possibilitando, por exemplo, a justificativa sobre o investimento.

Conhecer as ações a serem tomadas e os problemas a serem enfrentados na adoção de novas TI possibilita aos gestores a prevenção de problemas e a tomada de ações mais acertadas (FREITAS; RECH, 2003). Dessa forma, o entendimento consistente do processo de adoção tornase essencial para a eficácia dos processos decisórios relacionados à ADTI.

Para Lunardi e Dolci (2006), tradicionalmente a adoção das tecnologias mais modernas ocorre com mais rapidez entre as empresas de médio e grande porte, principalmente pelos altos investimentos exigidos. Entretanto, tem havido certa mudança de cultura entre os empresários das empresas de pequeno porte, o que resulta em esforço de aplicação de TI nas MPE. Adicionalmente, empresas fornecedoras de hardware e software têm identificado as MPE como um novo e atraente segmento de mercado.

Ao revisar a literatura sobre os modelos de adoção de tecnologias da informação em organizações, Oliveira e Martins (2011) afirmam que dentre as muitas teorias usadas na área de sistemas de informação, as mais usadas para análise da adoção de $\mathrm{TI}$ são: o technology acceptance model (TAM), a theory of planned behaviour (TPB), unified theory of acceptance and use of technology (UTAUT), diffusion of innovation (DOI) e a technology, organization and enviroment (TOE). Entretanto, salientam que apenas os modelos DOI e TOE são para análise em nível organizacional, sendo os demais (TAM, TPB e UTAUT) para análises no nível dos indivíduos. 
Ainda segundo Oliveira e Martins (2011), a DOI analisa a inovação organizacional a partir da influência das seguintes variáveis: características individuais, relacionadas à atitude dos líderes para mudança; características internas da estrutura organizacional, incluindo aspectos como centralização, complexidade, formalização e tamanho e características externas da organização, relacionadas ao entendimento da mesma como um sistema aberto. Sendo que esta teoria tem sido aplicada e adaptada em pesquisas na área de sistemas de informação (ROGERS, 1995).

Já o TOE identifica três aspectos no contexto organizacional que influenciam o processo pelo qual ocorre a adoção e implementação de uma inovação tecnológica, quais sejam: o contexto tecnológico, ou seja, aspectos internos e externos relacionados a tecnologias; contexto organizacional, refere-se a características como o escopo de atuação, tamanho e estrutura gerencial. Por fim, o contexto ambiental é analisado considerando a arena em que a empresa conduz o seu negócio, incluindo segmento de mercado, concorrentes e relações com o governo (BAKER, 2012).

O quadro 2 apresenta as principais teorias usadas para análise da adoção de tecnologias da informação, indicando seus principais autores e o nível de análise de cada umas delas.

Quadro 2: Teorias usadas na análise de adoção de TI.

\begin{tabular}{|l|l|l|}
\hline \multicolumn{1}{|c|}{ Teoria } & \multicolumn{1}{c|}{ Principais autores } & \multicolumn{1}{c|}{ Nível de análise } \\
\hline Teoria da Difusão da Inovação (DOI) & Rogers (1995) & Organizacional \\
\hline Modelo de Aceitação de Tecnologia (TAM) & Davis (1989) & Individual \\
\hline Teoria do Comportamento Planejado (TPB) & Ajzen (1991) & Individual \\
\hline $\begin{array}{l}\text { Teoria Unificada de Aceitação e Uso de } \\
\text { Tecnologia (UTAUT) }\end{array}$ & Venkatesh et AL (2003) & Individual \\
\hline Tecnologia, Organização e Ambiente (TOE) & Tornatzky e Fleischer (1990) & Organizacional \\
\hline
\end{tabular}

Fonte: Adaptado de Oliveira e Martins (2011)

\section{Micro e Pequenas Empresas e a Adoção de TI}

A adoção de TI em MPE começou a ser objeto de estudo na década de 1980, a partir do crescimento do uso de microcomputadores pessoais. Nessa época, as empresas de pequeno porte estavam relutantes em aplicar as tecnologias já em uso nas grandes corporações, pois possuíam pouco conhecimento sobre como seus negócios poderiam ser auxiliados pelas $\mathrm{TI}$ (LUNARDI; DOLCI; MAÇADA, 2010).

Conforme Ramos, Silva e Alvarega (2009), organizações têm incorporado TI em busca de diferenciais e vantagem competitiva. Entretanto, muitas MPE realizam investimentos sem a devida preparação de processos internos, dos próprios gestores e demais usuários. Assim, geram processos de mudança que conduzem, muitas das vezes, a uma subutilização desses recursos tecnológicos, sem alinhamento estratégico e muito menos adequação à realidade da empresa, gerando, em muitos casos, resultados inexpressivos.

A definição de pequenos negócios possui critérios variados ao redor do mundo. De acordo com o SEBRAE (2012), eles podem ser divididos por faixa de faturamento, em quatro segmentos, com exceção para o pequeno produtor rural. Essa segmentação considera os critérios da Lei 
Complementar 123/2006, chamada também de Lei Geral das Micro e Pequenas Empresas. Em resumo, os pequenos negócios são divididos da seguinte maneira:

- Empreendedor Individual - faturamento até $\mathrm{R} \$ 60 \mathrm{mil}$;

- Microempresa - faturamento até $\mathrm{R} \$ 360 \mathrm{mil}$;

- Empresa de Pequeno Porte - faturamento entre $\mathrm{R} \$ 360$ mil e $\mathrm{R} \$ 3,6$ milhões;

- Pequeno Produtor Rural - propriedade com até 4 módulos fiscais ou faturamento de até $R \$ 3,6$ milhões

O SEBRAE utiliza ainda, além do critério do Estatuto da Micro e Pequena Empresa, a definição do porte da empresa a partir do número de funcionários, principalmente nos estudos e levantamentos sobre a presença da micro e pequena empresa na economia brasileira. Abaixo são descritos esses números (SEBRAE, 2012):

- Microempresa: I) na indústria e construção: até 19 funcionários; II) no comércio e serviços: até 9 funcionários

- Pequena empresa: I) na indústria e construção: de 20 a 99 funcionários; II) no comércio e serviços, de 10 a 49 funcionários.

Para Lunardi e Dolci (2006), à medida que a aquisição de recursos de TI se torna mais acessível financeiramente, mais rápida é sua popularização, possibilitando também às micro e pequenas empresas utilizarem a informática nos seus processos de negócio. Fato ratificado pelo aumento do investimento em tecnologia nas MPE no final da década de 1990, quando o uso de microcomputadores cresceu entre 30 e 80\%. Tal aumento deveu-se, no caso das MPE, principalmente pela busca de vantagem competitiva, exigência de parceiros ou mesmo exigências legais.

Ainda segundo Lunardi e Dolci (2006), é possível analisar os fatores motivadores e inibidores da adoção de TI a partir de quatro construtos, descritos no quadro 3. Particularmente, concluíram em estudo com MPE que as pressões externas e o ambiente organizacional são os principais fatores de influência para este tipo de empresa, principalmente pela exigência dos negócios e pela concorrência acirrada.

Quadro 3: Fatores de influência na adoção de TI.

\begin{tabular}{|l|l|}
\hline \multicolumn{1}{|c|}{ Definição } & \multicolumn{1}{c|}{ Fonte } \\
\hline $\begin{array}{l}\text { Necessidade interna - a empresa adotou tecnologia em função do seu } \\
\text { crescimento ou para atender melhor as suas necessidades, garantindo dessa } \\
\text { forma o bom funcionamento da empresa. }\end{array}$ & $\begin{array}{l}\text { Fink (1988), Prates e Ospina } \\
(2004)\end{array}$ \\
\hline $\begin{array}{l}\text { Ambiente organizacional - a empresa adotou tecnologia porque percebeu que } \\
\text { possuía um ambiente favorável a sua utilização, com funcionários em } \\
\text { condições de utilizá-la e com uma estrutura organizacional adequada. }\end{array}$ & $\begin{array}{l}\text { Cragg e King (1993), Thong } \\
(2001), \text { Caldeira e Ward } \\
(2002)\end{array}$ \\
\hline $\begin{array}{l}\text { Pressões externas - a empresa adotou tecnologia em função da grande } \\
\text { concorrência existente, porque os concorrentes diretos têm adotado ou ainda } \\
\text { por influência de clientes, fornecedores ou o próprio governo. }\end{array}$ & $\begin{array}{l}\text { Cragg e King (1993), } \\
\text { Iacovou (1995), Grandon e } \\
\text { Pearson (2004) }\end{array}$ \\
\hline $\begin{array}{l}\text { Utilidade percebida - a empresa adotou tecnologia porque percebeu que ela } \\
\text { seria útil no seu dia-a-dia, melhorando a realização das tarefas e atividades da } \\
\text { empresa, aumentando a segurança, o controle e o atendimento aos clientes. }\end{array}$ & $\begin{array}{l}\text { Davis (1989), Iacovou } \\
\text { (1995), Grandon e Pearson } \\
\text { (2004) }\end{array}$ \\
\hline
\end{tabular}

Fonte: Lunardi e Dolci (2006). 
A adoção de tecnologias da informação em MPE gera diversos impactos positivos, como pode ser visto na Figura 4. De fato, quando ela é adotada corretamente, proporciona diversas vantagens, com destaque para a melhoria do atendimento aos clientes, qualidade na tomada de decisão, melhoria nos produtos e serviços e melhoria dos processos operacionais. A partir desses impactos, é possível concluir que a TI tem se tornado um fator estratégico e o seu gerenciamento torna-se essencial para o sucesso das empresas de pequeno porte.

Aumento de vendas

Reduçāo de pessoal

Reduçāo de estoques

Melhoria de produtos e serviços

Melhoria do atendimento ao cliente

Qualidade da tomada decisāo

Reduçāo de problemas operacionais

Aumento de lucros

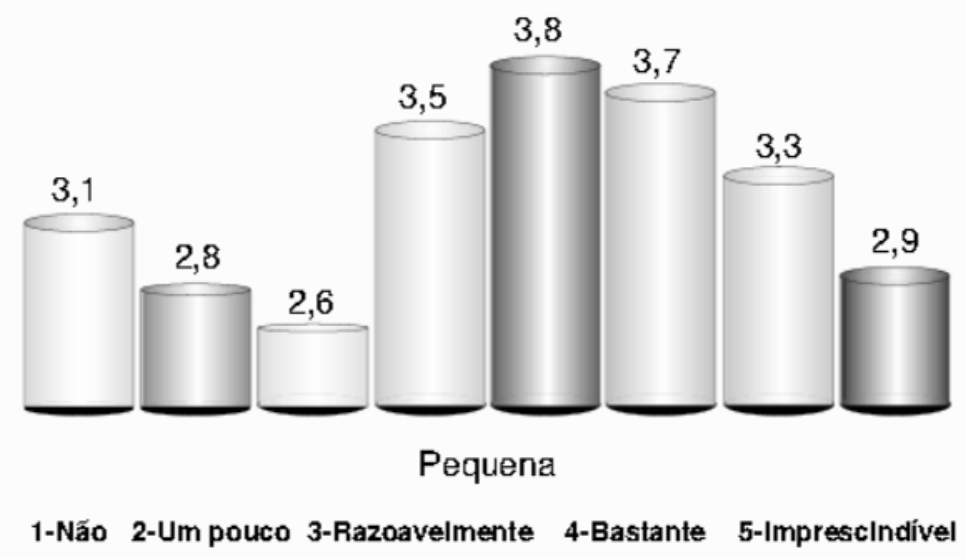

Figura 4: Impactos da TI em MPE. Fonte: Pesquisa "Perfil da empresa digital - 2002" (FIESP, 2003).

Considerados os benefícios impactados pelo uso de tecnologias da informação é essencial que estudos sejam conduzidos com esse intento. Entretanto, autores como Suhendra, Hermana e Sugiharto (2009) retratam que estudos relacionados a aplicação de $\mathrm{Tl}$ em pequenas empresas ainda são limitados quando comparados aos estudos em grandes organizações.

Sarosa (2007), em sua tese de doutorado, reconhece a adoção de TI em micro e pequenas empresas como um fenômeno complexo que possui três diferentes perspectivas de análise: individual, estrutural e processo interativo. A primeira focaliza nas ações dos indivíduos que iniciam o processo de adoção de TI. A segunda defende que o processo de inovação é determinado pelas características organizacionais e ambientais. Já a terceira, a escola do processo interativo, acredita que a adoção de inovações é resultado da interação entre indivíduos, organização e ambiente. Essas visões, a partir de perspectivas distintas, podem ser suportadas pela teoria contingencial do comportamento organizacional que reconhece que as organizações estão situadas em um ambiente e são constituídas por indivíduos que formam grupos a partir de suas interações.

A partir das considerações acima, que enaltece a relevância da temática, este estudo propõe a análise da adoção de TI em micro e pequenas empresas a partir do TOE, para tanto, propõe-se aqui a sua adaptação para que possa incorporar aspectos de tomada de decisão, essenciais, principalmente em MPE. 


\section{RESULTADOS}

\section{Modelo Proposto a Partir do Technology, Organization and Environment - TOE}

O modelo Technology, Organization and Environment - TOE foi proposto por Tornatzky e Fleischer (1990), onde os autores argumentam que o processo pelo qual uma organização adota e implementa inovações tecnológicas é influenciado pelo contextos tecnológico, organizacional e ambiental (Figura 5). O contexto tecnológico abrange as tecnologias internas e externas que são relevantes para a organização, que podem incluir tanto equipamentos quanto processos. O contexto organizacional refere-se às características e recursos da organização, incluindo seu tamanho, grau de centralização, grau de formalização, a estrutura gerencial, pessoas, e as relações entre os funcionários, entre outros elementos. O contexto ambiental inclui o tamanho e estrutura da indústria, os concorrentes, o contexto macroeconômico e do ambiente regulador (Tornatzky e Fleisher, 1990). Assim, esses três elementos influenciam o modo como uma organização identifica uma necessidade, procura, e adota uma nova tecnologia.

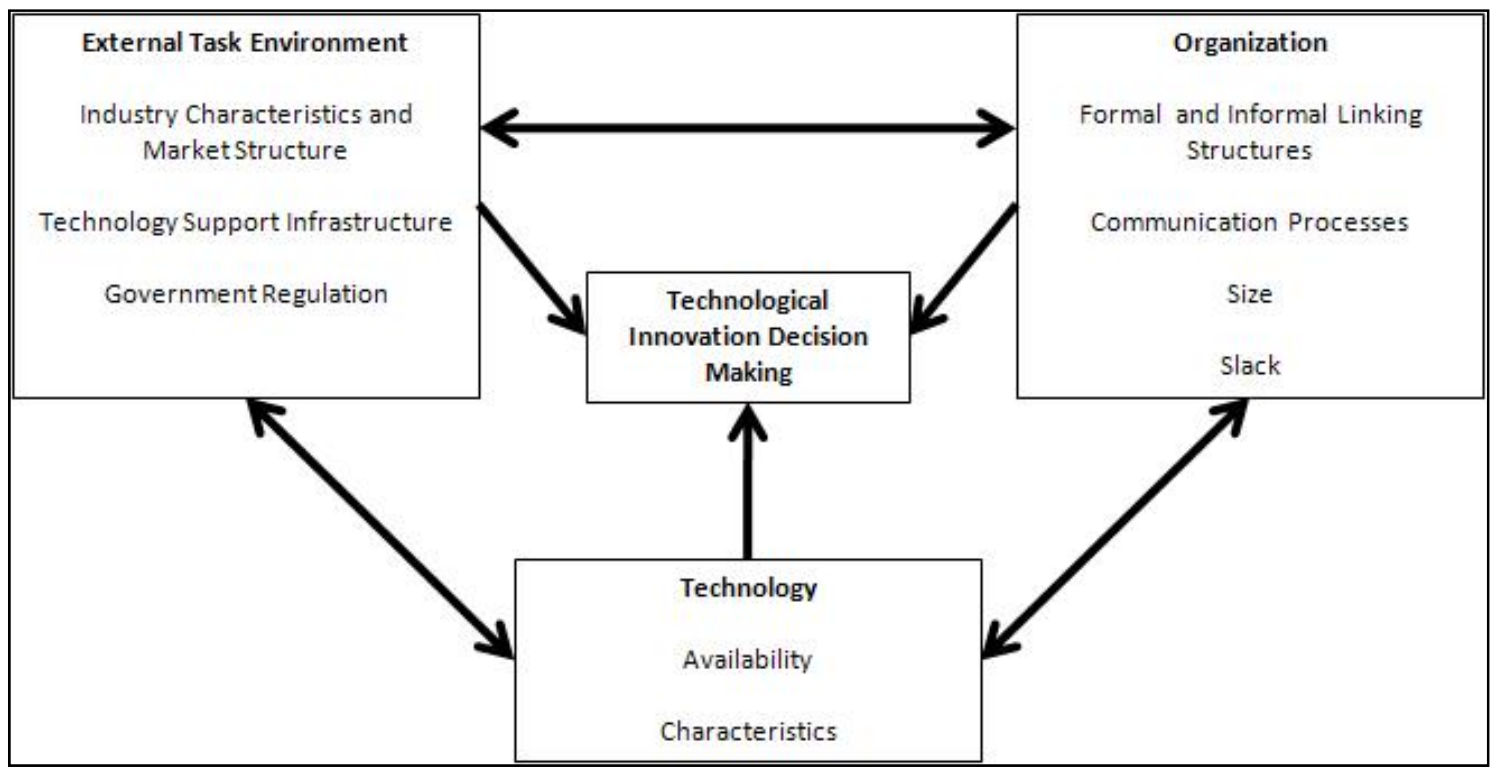

Figura 5: Modelo Technology, Organization and Environment - TOE. Fonte: Tornatzky e Fleischer (1990).

Este modelo tem sido testado e validado por vários estudos e é considerado um modelo aplicável à adoção e difusão de tecnologia, podendo ser usado inclusive para estudos em micro e pequenas empresas (RAMDANI; KAWALEK, 2008). Para Awa, Ukoha e Emecheta (2012), os contextos inerentes ao TOE podem ser caracterizados da seguinte forma:

- Contexto tecnológico: considera os aspectos de desenvolvimento tecnológico, ou seja, conjunto de fatores internos e externos à organização, inerentes à tecnologia. Ressalta-se que a análise de tal contexto deve considerar a percepção de vantagem competitiva a partir da tecnologia a ser adotada, compatibilidade técnica e organizacional, a complexidade e a curva de aprendizagem inerente a adoção de tecnologia e teste piloto. 
- Contexto organizacional: engloba a análise o escopo do negócio, o suporte da alta direção, a cultura organizacional. Além da complexidade da estrutura gerencial, medida em termos de centralização, formalização, verticalização, qualidade dos recursos humanos, tamanho e recursos disponíveis.

- Contexto ambiental: considera fatores facilitadores e inibidores para as operações organizacionais. Aspectos relevantes são: a pressão competitiva do setor, o escopo mercadológico de atuação, questões socioculturais, suporte governamental e a infraestrutura de suporte tecnológico.

Para Baker (2012), o modelo TOE representa um segmento de estudo, no contexto da área de pesquisa em inovação tecnológica. Afirma ainda que as pesquisas existentes têm demonstrado que o TOE possui aplicabilidade e poder explanatório em inúmeros contextos tecnológicos, industriais, em diferentes contextos culturais e de nacionalidade. Ademais, o TOE tem sido usado para estudos em diversos tipos de organizações, além de já ter sido testado em contextos da Europa, América e Ásia.

De acordo com Oliveira e Martins (2011), muitos autores utilizam o TOE em combinação com outras teorias, a exemplo da DOI, para um melhor entendimento das decisões acerca da adoção de tecnologias da informação. Entretanto, apesar de usar o termo decisão, não há aparente preocupação com os conceitos inerentes aos processos decisórios, lacuna que o modelo proposto no presente ensaio pretende minimizar, ao propor uma adaptação do modelo TOE, a partir da incorporação de aspectos conceituais de tomada de decisão.

Nesse sentido, o modelo proposto, ilustrado na figura abaixo, busca respaldo no modelo TOE, pois o mesmo, conforme ratifica Oliveira e Martins (2011), possui base teórica sólida e um suporte empírico consistente para estudos sobre adoção de tecnologia da informação. Entretanto, adiciona-se aqui reflexão crítica de Baker (2012) que observa a falta de estudos que contribuam para a evolução do modelo TOE. Desse modo, a intenção propositiva deste estudo tem inicio a partir da reformulação do aspecto central do modelo TOE, ou seja, o que é colocado originalmente como tomada de decisão para inovação tecnológica, terá, como pode ser visto na figura abaixo, um direcionamento específico para tomada de decisão no âmbito da adoção de tecnologia da informação. Além disso, o modelo aqui proposto busca também respaldo nos estudos sobre processo decisório para que possa contribuir para a evolução do entendimento teórico e empírico relativo à adoção de $\mathrm{TI}$.

A partir da nova perspectiva proposta, é essencial que sejam tratados conceitos inerentes aos estudos dos processos decisórios, principalmente quando considerado o contexto organizacional em análise.

De fato, a tomada de decisão normalmente busca a resolução de problemas ou o aproveitamento de oportunidades para geração de vantagens diante de mercados cada vez mais competitivos. Nesse contexto, o processo decisório também se aplica aos contextos de adoção de tecnologia da informação, em que se decide quais as melhores alternativas a serem adotadas por 
cada organização. As decisões envolvem a alocação de recursos e decidir significa efetuar escolhas diante de alternativas que combinem recursos e caminhos de ação a fim de atingir objetivos, considerando expectativas de resultados relacionados a cada alternativa. Assim, tais objetivos envolvem necessidades, desejos, valores, interesses e demais aspectos inerentes ao utilitarismo objetivo ou subjetivo de indivíduos ou grupos em uma organização (YU, 2011).

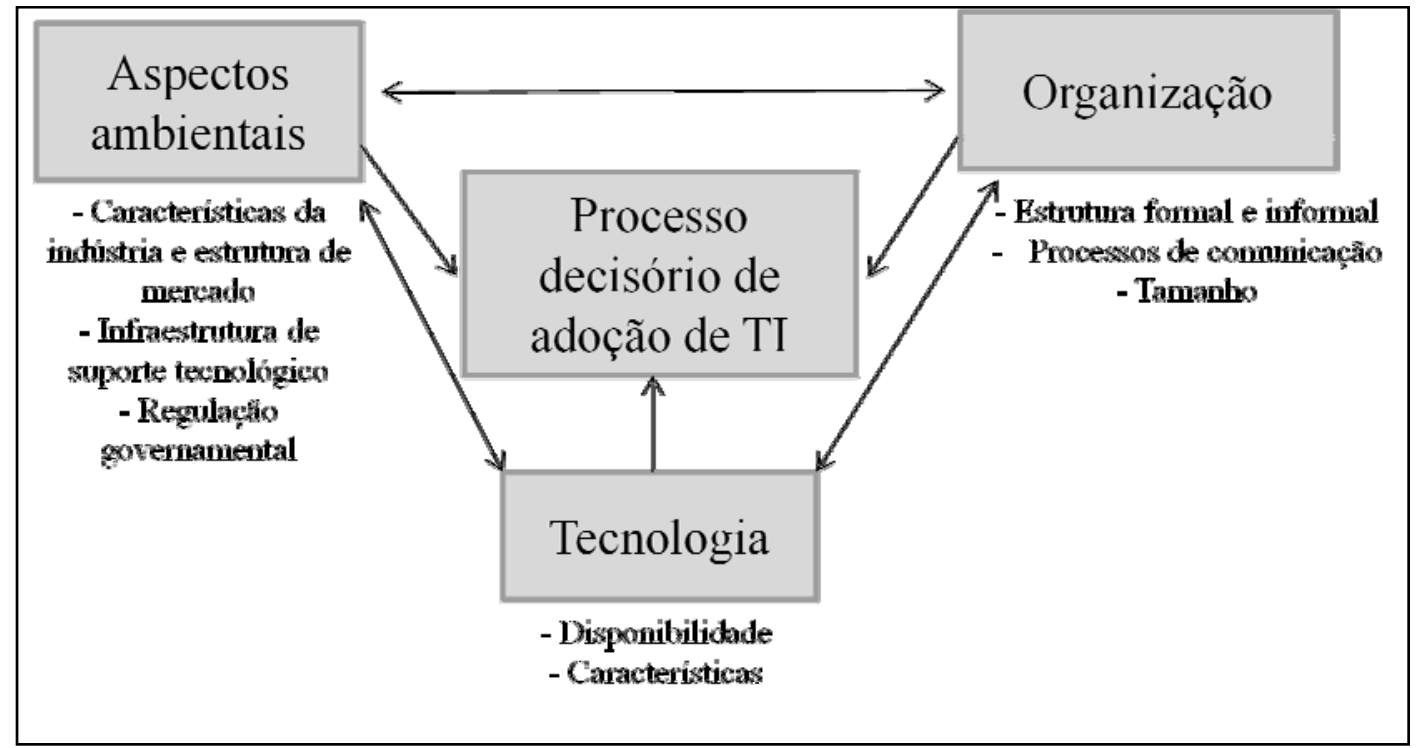

Figura 6: Modelo proposto para análise de adoção de TI. Fonte: Adaptado de Baker (2012)

O fluxo do processo decisório pode ser entendido a partir da figura abaixo que descreve as etapas inerentes à tomada de decisão, sendo essencial o seu entendimento para que o processo decisório inerente à adoção de tecnologias da informação seja tratado com a devida complexidade. Desse modo, inicialmente identifica-se a situação e entende-se os objetivos, a segunda consiste na identificação de alternativas possíveis ao problema ou oportunidade em análise. A terceira etapa é a decomposição do problema em modelagens que consideram estrutura e incertezas inerentes à situação, além das preferências e tolerância ao risco por parte do decisor, posteriormente escolhe-se a melhor alternativa, considerando o objetivo inicial. Há também a análise de sensibilidade a partir da comparação da alternativa escolhida diante de cenários. Por fim, a implementação da alternativa escolhida (RODRIGUES, 2009).

Segundo Ribeiro (2011) o processo decisório é amplo e complexo e envolve diversas etapas até ser concluído. Entretanto, tais etapas nem sempre são executadas na ordem previamente definida. Entretanto deve-se buscar o cumprimento das fases do processo para que a eficácia da decisão não seja comprometida.

Ainda para Ribeiro (2011), o processo decisório deve ser visto de forma sistêmica, pois as problemáticas organizacionais são complexas e possuem geralmente mais de uma causa e solução e envolvem diferentes setores da organização. Desse modo, a organização como um todo deve ser considerada e não apenas setores específicos que enfrentam problemáticas ou oportunidades de decisão. Ademais, é essencial que as organizações entendam a dinamicidade 
dos processos de decisão, pois as organizações estão em constante transformação e problemas e soluções devem ser constantemente revisitadas para análise e possível adequação de decisões.

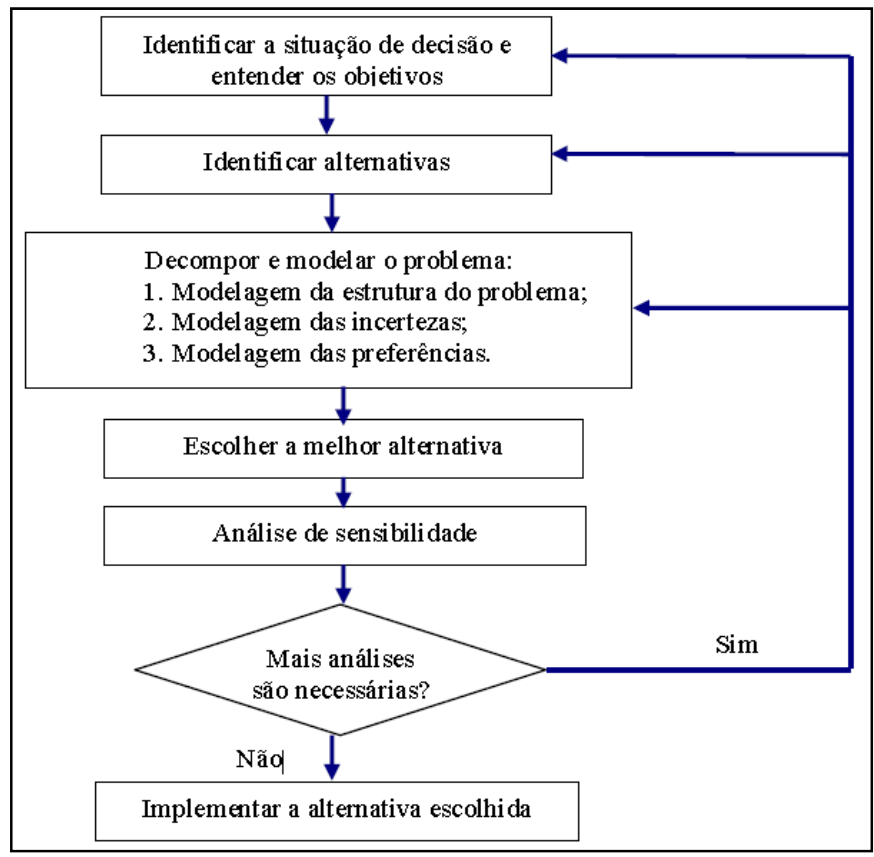

Figura 7: Etapas do processo decisório. Fonte: Clemen (1995 apud Rodrigues 2009)

Cunha e Soares (2010) complementam ao afirmar que tal dinamicidade, inerente aos processos de decisão, deve ser investigada também nos contextos de micro e pequenas empresas, diante da sua relevância no cenário econômico nacional. Desse modo, não se pode desconsiderar as complexidades encontradas no processo de tomada de decisão dessas empresas. Sendo micro ou pequenas, mas suas decisões não são micro e nem pequenas. Em alguns casos, a tomada de decisão pode afetar consideravelmente a continuidade das MPE, uma delas é a decisão sobre a adoção de tecnologias da informação.

\section{CONSIDERAÇÕES FINAIS}

A literatura evidencia que muitas empresas adotam $\mathrm{TI}$ a partir de diversos fatores, internos ou externos ao ambiente organizacional, e em processos distintos de incorporação. Segundo Lunardi, Dolci e Maçada (2010), as organizações que planejam efetivamente a aquisição de TI possuem maior possibilidade de potencializar o desempenho em mercados cada vez mais competitivos.

Por outro lado, a implantação de novas tecnologias pode proporcionar sucessos técnicos concomitantes a fracassos organizacionais (O'BRIEN, 2006). Tal problemática ocorre quando a tecnologia torna-se um fim em si mesma, ou seja, se for incorporada sem um processo consistente e consciente de adoção planejada de recursos de hardware, software e redes.

De fato, TI pode contribuir para a redução de custos, ganhos de produtividade, prospecção de novos mercados, facilidade de relacionamento com clientes e fornecedores, além de 
possibilitar melhor conhecimento do mercado de atuação e da conjuntura econômica. Entretanto, há heterogeneidade, principalmente quando trata-se de MPE, a exemplo das várias regiões do país, em que há diferenças com relação a fatores como qualificação, gestão, acesso a crédito e a novas tecnologias. (RAMOS; SILVA; ALVERGA, 2009).

Segundo Silva (2003), a problemática inerente à adoção de tecnologias da informação, principalmente em empresas de pequeno porte tem sido estudada por diversos autores, tanto da área de Administração quanto de Engenharia de Produção e permanece uma área crítica de pesquisa na literatura em Sistemas de Informação. Nesse contexto, é essencial revisitar e explorar teorias, renovando-as a partir de reflexões consistentes que levem a estudos exploratórios que retroalimentem tanto as práticas organizacionais quanto a literatura da área de sistemas de informação.

O presente trabalho buscou evidenciar a relevância de revisitar o tema da Adoção de $\mathrm{TI}$ e adicionalmente inovar a partir da reformulação de um modelo (TOE) para análise de micro e pequenas empresas. A partir da proposição, sugere-se que pesquisas futuras venham a aplicar o modelo proposto para que se verifique a riqueza exploratória de análise em organizações, principalmente as MPE, em virtude de sua relevância social e econômica, conforme dados apresentados neste trabalho.

A criticidade inerente a ADTI é reforçada quando se verifica nas MPE a falta de conhecimento técnico, falta de assessoria efetiva sobre os recursos tecnológicos necessários e a própria aquisição pouco acertada dos mesmos. Consequentemente, muitas empresas dessa natureza possuem resultados pouco expressivos e vivenciam a falta de alinhamento entre investimentos em TI e as estratégias da empresa (RAMOS; SILVA; ALVERGA, 2009).

Nesse sentido, o estudo evidenciou uma aparente falta de consideração de estudos sobre processo decisório para o entendimento da adoção de tecnologia da informação. A presente pesquisa, até o momento de sua publicação, não identificou autores que tratem do processo de adoção de TI a partir da consideração dos estudos de processo decisório. De fato, é evidente nos textos encontrados que a adoção de TI envolve decisões, entretanto, os estudos carecem da consideração de modelos de processo decisório efetivamente.

A partir da aparente ausência de processos claros a serem implantados nas organizações para a adoção de TI, principalmente que considerem modelos de processo de decisório e sejam adequados às micro e pequenas empresas, este ensaio intenciona a busca por minimizar a presença reduzida de modelos teóricos adequados a esse tipo de empresa.

\section{REFERÊNCIAS}

ALBANO, C.. Adoção de Novas Tecnologias da Informação: Um Estudo de Problemas e Ações nas Cooperativas Agropecuárias do Rio Grande do Sul. In: ENCONTRO DA ASSOCIAÇÃO NACIONAL DE PÓS-GRADUAÇÃO E PESQUISA EM ADMINISTRAÇÃO, 25. Anais. Campinas: ANPAD, 2001.

AMARAL FILHO, J.. É negócio ser pequeno, mas em grupo. In. ROVERE, R. L.. Painel Micro, Pequenas e Médias Empresas. Rio de Janeiro: UFRJ, 2012. 
AJZEN, I.. The theory of planned behavior. Organizational Behavior and Human Decision Processes, v.50, p.179-211, 1991.

AUDY, J; BRODBECK, A.. Sistemas de Informação: planejamento e alinhamento estratégico nas organizações. Porto Alegre: Bookman, 2003.

AWA, H. O.; UKOHA, O.; EMECHETA, B. C.. Integrating TAM and TOE Frameworks and Expanding their Characteristic Constructs for E-Commerce Adoption by SMEs. Proceedings of Informing Science \& IT Education Conference (InSITE) 2012.

BAKER, J.. The Technology-organization-enviroment Framework. In: Information Systems Theory: explaining and predicting our digital society. Springer Science + Business Media, 2012.

BATISTA, E.. Sistemas de Informação: uso consciente da tecnologia para o gerenciamento. São Paulo: Saraiva, 2004.

BELTRAME, M.; MAÇADA, A.. Validação de um Instrumento para medir o Valor da Tecnologia da Informação (TI) para as organizações. Organizações em contexto, v.5, n.9, p.1-23, 2009.

CORDENONSI, J. L.. Alinhamento das estratégias de TI e de negócio: as melhores práticas utilizadas pelos bancos privados brasileiros. In: ALBERTIN, A. L.; ALBERTIN, R. M. M.. Tecnologia da informação: desafios da tecnologia de informação aplicada aos negócios. São Paulo: Atlas, 2005.

CUNHA, A.; SOARES, T.. Aspectos relevantes do planejamento no crescimento das micro e pequenas empresas (MPE). Revista da Micro e Pequena Empresa, v.4, n.3, p.15-39, 2010.

COMPUTERWORLD. Disponível: <http://computerworld.uol.com.br/especiais/2012/10/29/empresasbrasileiras-vaos-gastar-us-134-bilhoes-em-ti-em-2013>. Acesso: 3 nov 2012.

DAVIS, F. D.. Perceived usefulness, perceived ease of use, and user acceptance of information technology. MIS Quarterly, Minneapolis, v.13, n.3, p.318-339, 1989.

FIESP. Pesquisa "Perfil da empresa digital. Disponível: <http://www.sindlab.org/download_up/idigital2003.pdf>. Acesso: 4 nov 2012.

FREITAS, H.; RECH, I.. Problemas e ações na adoção de novas tecnologias de informação. Revista de Administração Contemporânea, São Paulo, v.7, n.1, p.125-149, 2003.

GUERRA, A.; CAPOVILLA, I.; THIENNE, R.. O processo de software e seus atores sociais. In: WORKSHOP UM OLHAR SOCIOTÉCNICO SOBRE A ENGENHARIA DE SOFTWARE - WOSES, 1. Anais. Rio de Janeiro: PESC/COPPE/UFRJ, 2005.

LUNARDI, G.; DOLCI, P.. Motivadores e inibidores da adoção de tecnologia de informação (TI) nas micro e pequenas empresas. In: ENCONTRO NACIONAL DE ENGENHARIA DE PRODUÇÃO, 26. Anais. Fortaleza: ABEPRO, 2006.

LUNARDI, G.; DOLCI, P.; MAÇADA, A.. Adoção de tecnologia de informação e seu impacto no desempenho organizacional: um estudo realizado com micro e pequenas empresas. Revista de Administração da USP, v.45, n.1, p.5-17, 2010.

MEIRELLES, F.. Informática: novas aplicações com microcomputadores. 2 ed. São Paulo: Makron Books, 2003.

O'BRIEN, J.. Sistemas de Informação e as decisões gerenciais na era da internet. 2 ed. São Paulo: Saraiva, 2006.

OLIVEIRA, T.; MARTINS, M.. Literature Review of Information Technology Adoption Models at Firm Level. The Electronic Journal Information Systems Evaluation, v.14, n.1, p.110-121, 2011. 
RAMDANI, B.; KAWALEK, P.. SMEs \& IS innovations adoption: A review \& assessment of previous research. Revista da Academia Latinoamericana de Administracion, v.39, p.47-70, 2008.

RAMOS, A.; SILVA, E.; ALVERGA, P.. O papel estratégico da TI nas micro e pequenas empresas. Natal: SEBRAE/RN, 2009.

RIBEIRO, D.. Planejamento estratégico e processo decisório em micro e pequenas empresas. 2011. 52 f. Monografia (Bacharelado em Administração) - Universidade de Brasília, Brasília, 2011.

RODRIGUES, F.. Dinâmica do processo decisório em equipe: análise temporal-ambiental. 2009. 272 f. Tese (Doutorado em Administração) - Universidade de São Paulo, São Paulo, 2009.

ROGERS, E. Diffusion of innovations. 4ed., New York: Free Press, 1995.

SANTOS, E.. Fatores condicionantes da adoção de tecnologias da informação nas organizações In: CATI 2005 - CONGRESSO ANUAL DE TECNOLOGIA DE INFORMAÇÃO, 2. Anais. São Paulo: 2005.

SANTOS JUNIOR, S.; FREITAS, H.; LUCIANO, E.. Dificuldades para o uso da tecnologia da informação. RAE Eletrônica, v.4, n.2, 2005.

SAROSA, S.. The Information Technology Adoption Process within Indonesian Small and Medium Enterprises. 2007. Tese (Doutorado Tecnologia da Informação) - University of Technology Sydney, 2007.

SEBRAE. Critérios e conceitos para classificação de empresas. Disponível:

<http://www.sebrae.com.br/uf/goias/indicadores-das-mpe/classificacao-empresarial>. Acesso: 4 nov 2012.

SILVA, E.. Adoção de tecnologias de informação nas micro e pequenas empresas fornecedoras da Petrobrás no Rio Grande do Norte. Dissertação (Mestrado em Engenharia de Produção) - Universidade Federal do Rio Grande do Norte, 2003.

SUÁREZ, P.; SILVA, Y.; SOUZA, S.. Sistemas de Apoio À Decisão para a Adoção de Tecnologia de Informação - a Construção de um Protótipo. In: SIMPÓSIO DE EXCELÊNCIA EM GESTÃO E TECNOLOGIA, 8. Anais. Resende: Associação Educacional Dom Bosco, 2011.

SUHENDRA, E.; HERMANA, B.; SUGIHARTO, T.. Behavioral Analysis of Information Technology Acceptance in Indonesia Small Entreprises. In: ANADOLU INTERNATIONAL CONFERENCE IN ECONOMICS. Anais. Turkey: Anadolu University,2009.

TORNATZKY, L.; FLEISCHER, M.. The Processes of Technological Innovation. Massachusetts: Lexington Books, 1990.

TAPSCOTT, D.. O que esperar do mundo digital. HSM Management, São Paulo, v.2, n.12, p.132-136, 1999.

VENKATESH, V. et al.. User acceptance of information technology: toward a unified view. MIS Quarterly, Minneapolis, v.27, n.3, p.425-478, 2003.

YU, A.. Tomada de decisão nas organizações. São Paulo: Saraiva, 2011. 